\title{
Governance in Blockchain Technologies \& Social Contract Theories
}

\author{
Wessel Reijers, ${ }^{* \dagger}$ Fiachra O'Brolcháin, ${ }^{\dagger}$ Paul Haynes ${ }^{\S}$
}

\begin{abstract}
This paper is placed in the context of a growing number of social and political critiques of blockchain technologies. We focus on the supposed potential of blockchain technologies to transform political institutions that are central to contemporary human societies, such as money, property rights regimes, and systems of democratic governance. Our aim is to examine the way blockchain technologies can bring about - and justify - new models of governance. To do so, we draw on the philosophical works of Hobbes, Rousseau, and Rawls, analyzing blockchain governance in terms of contrasting social contract theories. We begin by comparing the justifications of blockchain governance offered by members of the blockchain developers' community with the justifications of governance presented within social contract theories. We then examine the extent to which the model of governance offered by blockchain technologies reflects key governance themes and assumptions located within social contract theories, focusing on the notions of sovereignty, the initial situation, decentralization and distributive justice.
\end{abstract}

\section{Introduction}

The Blockchain, the technological innovation underpinning the familiar cryptocurrency Bitcoin, is increasingly the topic of academic and public debate. In this paper, we aim to examine the ways in which blockchain technologies can produce models of governance and how these models of governance are justified. We do so by exploring similarities between core design features of the Blockchain, the main ideas about governance that persist in the blockchain community and essential aspects of prominent social contract theories. We do not intend to construct a conclusive comparison between models of government offered by social contract theories and blockchain technologies, but rather to identify points of convergence and divergence that enable us to indicate points of departure for political critiques of the technology.

Blockchain technology, first applied in the design of Bitcoin in 2008, emerged from a movement of anarchists, computer scientists and crypto-enthusiasts who saw the potential of the technology as a breakthrough in the long-awaited realization of an old "cypherpunk" dream of money that is free from the control of the state and other third parties, such as commercial banks; ${ }^{1}$ however, blockchains offer technological possibilities far beyond new ways of issuing money. They also offer scope for rethinking political organization, including enabling novel ways of creating, managing and maintaining systems of voting rights, property rights and other legal agreements. We refer to the process by which blockchains enable such

\footnotetext{
${ }^{\dagger} W$. Reijers (wreijers@adaptcentre.ie) is a PhD researcher at the School of Computing, Dublin City University

${ }^{\dagger} \mathrm{F}$. O'Brolcháin (fiachra.obrolchain@dcu.ie) is a postdoctoral researcher at the Institute of Ethics, Dublin City University

${ }^{\S}$ P. Haynes (paul.haynes@rhul.ac.uk) is lecturer at the School of Management, Royal Holloway, University of London * 3HrFGw5nuBup39tzvQT5reEF5gdtx8fDGw
} 
systems as "blockchain governance," which is constitutive of a broader political theme termed "blockchain government." 2

Our paper contributes to a growing body of political and sociological reflections on blockchain technologies in which the design and application of its technology is linked to ideas of political organization. Kostakis and Giotitsas (2014: 437) argue that Bitcoin "as a piece of software is imbued with ideas drawn from a certain political framework." 3 Such a political framework, Barton (2015) argues, challenges the instrumentalist idea of technical "neutrality" of Bitcoin, ${ }^{4}$ a claim he supports with ethnographic findings indicating biases present in the design of the technology itself. Golumbia (2015: 128) is more explicit, stating that networks built on the Blockchain represent a political framework that is "profoundly antidemocratic" and serves "a neo-liberal agenda." In addition, some scholars specifically focus on philosophical ideas of political organization that can be traced in the technological design of the Blockchain. For instance, Dupont (2014: 8) argues that cryptographic code can "stand in" for humans and that the Blockchain can be regarded as a powerful "ordering machine" in the modern "control society." " 6 Linking Bitcoin to political philosophy, Kavanagh and Miscione (2015: 8) draw the connection between the Blockchain and the Leviathan, as conceptualized in the work of Thomas Hobbes, as the enforcer of the social contract. ${ }^{7}$ More specifically, Dupont and Maurer (2015) argue that the Blockchain conjoins "two of the central legal devices of modernity: the ledger and the contract." ${ }^{8}$ Our paper aims at contributing to these philosophical debates by exploring philosophical ideas common to both the Blockchain and classical social contract theories.

We base our argument on the social contract theories of Hobbes, Rousseau, and Rawls, and on central texts produced by, and widely circulated within, the blockchain developer community. Notably, we focus on writings about the Ethereum platform. Ethereum is a nonprofit organization with the key objective stipulated as: "promotion of developments of new technologies and applications, especially in the fields of new open and decentralized software architectures." ${ }^{9}$ Its character, as a platform for the advocacy and development of blockchain applications that tries to engage the wider community of developers, users and enthusiasts, makes it a valuable source for investigating how principles of political organization are discussed in the context of blockchain technologies. As in any community, proponents of blockchain technology express a diversity of views representing a variety of perspectives; however, the values that unite the Ethereum community can be drawn from a number of its key texts. For our case study, these include white and yellow papers (Buterin, 2013; Wood, 2014) and communications from key individuals, organizations and other members of the Ethereum community (including interviews, articles, mission statements, wiki, blog and forum postings).

Our inquiry is guided by two distinct research objectives. Firstly, we investigate the extent to which justifications of blockchain governance offered by the Ethereum community reflect justifications of governance offered by social contract theories. Secondly, we investigate the extent to which the model of governance offered by blockchain technologies reflects the models of governance offered by prominent versions of social contract theory. We start by outlining the principles of governance applied in the Blockchain, focusing on two of its key features: its nature as a public ledger, and its capacity to decentralize the enforcement of contracts. We then compare justifications offered for blockchain governance with justifications for governance offered by the social contract theories of Hobbes, Rousseau and Rawls. Finally, we trace similarities between the models of governance offered by these theories and the model of governance enabled by blockchain technologies. 


\section{How Blockchain Technologies Can Shape Governance}

We start our investigation by exploring the way blockchain technologies are able to configure specific forms of political organization. In order to do so, we focus on a paradigmatic instance of a software project utilizing blockchain technology: Ethereum. Ethereum was chosen as a case study because it matches a number of relevant criteria, including its technological scope and the engagement with political ideas by its community of practitioners. It aims at implementing the paradigm of the Blockchain "coupled with cryptographically-secured transactions" in a "generalized manner." 10 This suggests that it attempts to generate a software standard (like an e-mail protocol) for any kind of decentralized blockchain application, which could range from another cryptocurrency to applications for managing "smart-contracts" like blockchain-instigated civil marriage contract, ${ }^{11}$ property contracts and financial instruments. ${ }^{12}$

The Blockchain can be described as a public record of time-stamped transactions that is reinforced by the computational efforts of the decentralized network of 'miners' (people controlling computational nodes that are validating transactions). This public record is commonly referred to as the "universal" or public ledger. Core features of blockchain design that are relevant for our analysis are: (i) its nature as a digital, public ledger through which people contract with one-another; and, (ii) its decentralized enforcement of validated transactions or contracts by means of computational scrutiny. Any blockchain consists of time-stamped "blocks," which are collections of the validated transactions in the system within a certain timeframe (every 10 minutes in the case of Bitcoin). All transactions made within a blockchain are available to public inquiry, from the "beginning of time" (when the first block was time-stamped) until the current moment. In theory at least, this means that all the entities interacting with a certain blockchain application can own a copy of the public blockchain and control the validity of new interactions. Thus, so-called "smart contracts" in the given blockchain can be publicly validated and can be enforced by a decentralized network of nodes; which can in theory include all the users of the blockchain.

The objects that are transacted through a blockchain need not be quantities of money, as is the case with Bitcoin, but can also be texts or certain rule-based agreements. Aspects of governance such as property rights regimes, insurance contracts and even so-called "decentralized autonomous organizations" (DAOs) - organizations such as companies or government institutions that are managed by means of decentralized, blockchain-based interactions - can be (re)organized and managed through blockchain technologies. ${ }^{13}$ Property rights can for instance be organized on a blockchain in the context of the Internet of Things (IoT). In this context, physical devices that are connected to the Internet would require identification of their owner in order to be used, with the ownership rights of each specific device stored on a blockchain (Wright and De Filippi 2015: 15). This is an important innovation because, as Dupont and Maurer (2015) argue, blockchain technologies differ from traditional social systems that validate, maintain and enforce contracts between people (e.g. accountancy and legal systems), because "cryptocontracts tend to build social and functional properties within the system." In other words, where lawyers and judges are needed to enforce legal regulations and notaries are needed to validate certain legally binding contracts, the blockchain allows for the validation of smart contracts and their enforcement in its own right without the necessity for arbitrating third parties. Because of these features, developers of the Ethereum platform argue that the blockchain can function as a legal framework able to serve as the basis for online interactions of any kind, claiming that: "Ethereum is a new kind of law." ${ }^{14}$ This implies that in contrast with conventional contract laws, which are necessarily 
coupled with their human validators and enforcers, blockchain technologies are capable of establishing and maintaining forms of political organization that are (at least in the virtual realm) self-sustaining.

As Dupont and Maurer (2015) argue, the public ledger renders social interactions that are recorded on the ledger visible to everyone in the system (both human and artificial agents in the case of the Ethereum ledger), which consequently renders them auditable. Moreover, the decentralized enforcement of smart contracts "dematerializes" or rather depersonalizes the auditing authority: it eradicates the need for human arbitrators such as notaries or accountants. To understand how blockchain technologies enforce "smart contracts" as opposed to how traditional contracts are enforced, we need to clarify both terms. Traditional contracts can be described as textually expressed voluntary agreements between two or more contracting parties that require human arbitration to be validated, audited and enforced. A smart contract is defined by Buterin (2016) as "a mechanism involving digital assets and two or more parties, where some or all of the parties put assets in and assets are automatically redistributed among those parties according to a formula based on certain data that is not known at the time the contract is initiated." 15 Thus, on the one hand we can say that clauses sanctioned by two parties in conventional contracts are textually defined and do not directly bind the contracting parties because a third, arbitrating human party is necessary to ensure the validity and enforcement of the contract. On the other hand, a smart contract implies that all the contractual clauses are machine-readable and can be made binding by means of computational scrutiny, without human interference. As Dupont and Maurer (2015) put it, the smart contract "replaces the difficult social and psychological work of contracting with self-executing code." We would slightly nuance this claim by stating that a significant part of the "work of contracting" remains embedded in social interactions, namely the act of consenting to a specific contractual reality. The aspects that are delegated to the technology are the validation, storing and enforcement of the contractual clauses.

The characteristics of blockchain technologies, as described earlier, seem to support the claim that they could, in many circumstances, mimic institutional processes that enable society governance, such as currency systems (as Bitcoin demonstrates), property regimes and even democratic voting processes. Whether such institutional processes on the blockchain can be part of a "social contract" similar to the social contract as understood in the philosophical tradition, remains, however, an open question. In the following section, we explore the extent to which the "social contract" of blockchain governance reflects aspects of the social contract that structures the basis of governance as theorized by some of the most prominent thinkers in the philosophical tradition.

Before we proceed with this inquiry, we need to clarify two important issues. First of all, we need to clarify the meaning of "social contract" vis-à-vis the notions of contract and smart contract discussed earlier. In philosophical writings, the concept of the social contract is used in two distinct traditions: one identified by Skyrm (1996: ix) as focusing on "what sort of contract rational decision makers would agree to in a preexisting "state of nature", and another that aims to explain how the implicit social contract that creates society has evolved and may continue to evolve in the future. ${ }^{16}$ In this paper, we limit our focus to an understanding of the social contract as it is used within the first of these traditions, i.e. conceptualizing the social contract as a method for justifying political principles by appeal to an agreement made in an initial situation by people who are (broadly speaking) presupposed to be equal, rational, and autonomous. 
This notion of the social contract is one of the most significant contributions of Western liberal political philosophy. Its lineage can be traced back to Thomas Hobbes (1651), JeanJacques Rousseau (1762), and John Rawls (1971). ${ }^{17,18,19}$ We acknowledge that by focusing on these three thinkers our account of the social contract tradition will remain incomplete, not least because it excludes other notable contributors (e.g. Locke, Gauthier, Schmitt). Nevertheless, we argue that within the scope of this paper the three thinkers selected afford a discussion of the most significant aspects of social contract theories. Social contract thinkers were attempting to justify government - arguing that governments were legitimate if they were deemed to be the creations of autonomous individuals contracting together. Governments are, in this way, conceptualized as systems designed to protect certain central aspects of human existence - life for Hobbes, a substantive conception of liberty for Rousseau, and justice as fairness for Rawls. The perception that governments provide such protections is considered sufficient to legitimize the loss of certain rights and the allocation of power to specific supra-individual structures, such as constitutional monarchies or parliamentary democracies. In the sense that social contract theories do not merely explain why people agree to form a government to inaugurate certain political principles but also stipulate what these principles (ideally) are, they therefore offer certain abstract models of governance. The models of governance presented by social contract theories can be obtained by looking at how they postulate the process through which people collectively contracting are able to overcome the hypothetical initial situation.

Additionally, we need to explain why we believe a discussion of social contract theories could advance our understanding of how blockchain technologies configure forms of governance. In the context of some of the core writings on blockchain technologies, this can be explained with reference to the myriad occasions on which the social contract is mentioned (see e.g. Buterin 2014; Chuen 2015; Wood 2014). In these writings, the "social contract" is commonly conceptualized as the rule-based, distributed system containing the public ledger on which smart contracts are based. The crucial difference between smart contracts and the social contract in these writings is therefore that smart contracts are protocols enforcing specific contractual agreements that are built on top of and conditioned by the underlying system (such as Ethereum), which in its entirety can be referred to as "the social contract." The social contract for blockchain technologies can thus be understood as the underlying model for the governance of blockchain-based interactions.

However, it is not at all self-evident to claim that the notion of a social contract as used in the context of blockchain governance can be said to reflect, or possibly even embody, aspects of the models of governance contained in philosophical social contract theories. To support this claim, we assert, as Golumbia argues, that technologies such as the Blockchain are not neutral but might be "deeply political" (2015: 118). In philosophy, scholars such as Ihde and Winner have shown that technologies can embody normative and political ideas. ${ }^{20,21}$ Georg Simmel offers a forceful example of an analysis based on this assumption in his work The Philosophy of Money. ${ }^{22}$ Simmel argues that the empirical realizations of money (coins, credit) move towards a conceptual ideal of "pure money" (1900: 508), which is the expression and embodiments of his conceptual construct of exchange as a condition of economic value (1900: 79-87). Even though the conceptual ideal of pure money is unattainable in empirical reality, ${ }^{23}$ it functions as an actual force that guides the design of our monetary system. Similarly, we could argue that even though the abstract models of governance offered by social contract theories are postulated as hypothetical ideals, they also inform real-world political constructs. As such, conventional political constructs such as constitutions in many ways reflect aspects 
of ideal models of governance explicated by social contract theories. Expanding on this idea suggests that technologies such as the blockchain might similarly reflect aspects of social contract theories, a view we will examine in the following sections.

\section{The "Initial Situation" and Justification of Blockchain Governance}

In this section we examine the extent to which the justification for governance enabled by blockchain technologies (blockchain governance) reflects one or more of the accounts of justification offered by social contract theories. The social contract theories of Hobbes and Rousseau aimed to justify the existence of a legitimate government by postulating a conceptual "state of nature," or initial situation, populated by somewhat isolated individuals of roughly equal power and capacity. Rawls constructs a hypothetical "original position of equality" (1971: 11), which corresponds to the state of nature but puts the contracting individual behind a conceptual "veil of ignorance." The initial situation serves as a rationale for such isolated individuals to agree to collectively relinquish (some of) their individual rights for the sake of forming a supra-individual structure of government. For Hobbes, a core feature of the state of nature is that it results in a high level of uncertainty for its inhabitants, ${ }^{24}$ implying that individuals are unable to reach agreement on certain issues because they cannot trust that all parties involved will honor the agreement. This leads to the situation described by Chung as a constant potential for a "war of every man against every man" (Chung, 2015: 485), a state of affairs undesirable for the individuals living in this situation, which provides them with the justification to form a government.

Rousseau's social contract theory is based on a notion of "initial situation" that is significantly different from that of Hobbes. Rousseau viewed the state of nature, the precivilized state of human society without government, as a peaceful, idyllic situation. It is only with the rise of institutions such as private property and money that an undesirable state of affairs arises. ${ }^{25}$ The institutions created by people have corrupted society and have instantiated unjust forms of inequality between people. This institutional reality is what serves as Rousseau's initial situation, which should be overcome by means of a specific social contract. In a similar vein, Rawls's "original position" is meant to serve as a rationale for the contracting individuals to engage in a social contract able to promote justice as fairness for all its contracting parties. Behind the veil of ignorance, contracting parties are unaware of their own position (as defined by gender, race, class etc.) vis-à-vis the positions of the other contracting parties. Because an individual is placed behind the conceptual veil of ignorance, she is uncertain about her eventual position once the social contract is in place. This provides for the rationale and the justification for the individual to agree to a social contract that is as fair as possible for all contracting parties.

Before addressing the parallels, we need to acknowledge that the philosophical underpinning of blockchain governance differs from that of the social contract tradition, by being strongly aligned to anarchist and libertarian theories of social order, with many thinkers within this tradition, such as Nozick and Proudhon, argue strongly against the notion of a social contract. ${ }^{26,27}$ Nevertheless, we will indicate below that some essential aspects of the justification for blockchain governance show significant similarities with justifications offered by social contract theories. It should be noted that it is impossible to refer to single scholars or single works in order to capture the established justification of blockchain governance. As such, any absolute claim of defining the "blockchain ideology" can be greeted with skepticism. However, we contend that by studying the core texts that support its most 
prominent instantiations, as exemplified by Ethereum in our research, we can at least construct a coherent account of the justification offered for blockchain governance.

To what extent can we say that justifications of blockchain governance reflect aspects of the types of justification for governance as offered by Hobbes, Rousseau or Rawls? The Ethereum community provides illuminating justifications of the two core features of the blockchain we discussed earlier: of the public ledger and the decentralized system of enforcement of transactions. In the Ethereum white paper, it is argued that these two features solve two important political enigmas: of people corrupting systems by means of fraud and counterfeiting and the freeing of human beings from central political powers such as states and banks. ${ }^{28}$ At face value, this outlook ties in with anarchist and libertarian critiques of authority. Such critiques claim that centralized powers like states and banks are easily corrupted and that groups of individuals are able to organize themselves in sophisticated ways in the absence of third-party institutions. As an alternative form of governance, proponents claim that through blockchain technologies autonomous individuals are capable of creating a self-governing community (or multiple communities) with enforceable rules of interaction without the requirement of any centralized (hierarchical) power structures.

In spite of these ideological tensions, some striking similarities between the justification of blockchain governance and the justification of governance offered by social contract theories can be observed. First of all, similar to the initial situation as conceptualized by Rousseau, blockchain governance is justified against the idea of an initial "pre-blockchain" society. Roio argues that events such as the blockade of payments to Wikileaks by the US government and major payment companies in 2010 have been important enablers of theme he identifies as the "cypherpunk imagination," ${ }^{29}$ justifying the use of Bitcoin as an alternative payment system. As such, blockchain governance is justified by reference to an idealized initial, undesirable situation that is defined by the contemporary institutional reality of centralized institutions, which are subject to human arbitration. Moreover, just as Rawls's original position can be used as a justification of net neutrality, as Schejter and Yemini argue, ${ }^{30}$ blockchain governance can be justified with reference to a notion of "neutrality." In this respect regard, the technology itself functions as a "veil of ignorance" in that it is unable to discriminate between its users, in contrast to conventional institutions.

However, the justification of blockchain governance differs significantly from the justifications offered by Rousseau and Rawls in two ways. Firstly, even though people interacting through blockchain applications could theoretically operate through a "veil of ignorance"-in the sense that they could enjoy a high level of pseudonymity and the technology would be structurally incapable of discriminating against them on the basis of who they are-power is still divided unequally. This is the case because, as the definition of the smart contract reveals, relations between contracting parties are defined in terms of digital assets (for instance in the form of a bet, with person A betting $x$ amount of Bitcoins and person B $y$ amount on the same predicted outcome of an event). Therefore, a situation of neutrality as defined by Rawls's original position would be unattainable in the blockchain, because power-relations are always already predefined in the public ledger. Secondly, the conception of human nature guiding Rousseau's justification for the social contract differs strongly with the conception of human nature offered for the justification of blockchain governance. Rousseau views human society as naturally peaceful and friendly, but argues that it has been corrupted by civilization. The blockchain community, in contrast, envisions human nature and especially the notion of "trust" in humans as the corrupting factors in contemporary civilizations. As O'Dwyer argues, the claim is made that trust in humans is undesirable and 
should be made redundant by replacing it with a different kind of trust, namely the "trust in the code." 31

These aspects of the justification of blockchain governance lead us to consider the justification made by Hobbes for the social contract. As Kavanagh and Miscione argue, a conceptual situation similar to the circumstances described by Hobbes is outlined by Nakamoto in his white paper on Bitcoin, framing the issue as a problem of "costs and payment uncertainties" between merchants and customers, ${ }^{32}$ which causes distrust (understood as distrust between humans). Nakamoto's account is similar to the one offered by Hobbes - both accounts envision the potential for corrupt behavior in a situation of uncertainty. This presupposition is consistent with the negative view of human nature expressed by Hobbes, which accepts that humans will engage in corrupt behavior if it serves their self-interest. A similar assumption seems to underlie the rationale for replacing trust in potentially corrupt humans by the incorruptible code of the blockchain.

Additionally, as Rawls (1971: 238) and Chung (2015: 490) argue, the initial situation described by Hobbes in the context of his mechanical worldview can be understood as a game-theoretical problem. The equilibrium of a war of every man against every man can be expressed in game-theoretical terms, just as its solution, which is the social contract as described by Hobbes. Similarly, both the initial situation (the pre-blockchain world) and blockchain governance are commonly grounded in a game-theoretical understanding of the world. As Buterin argues: "the same game theory that is the reason that you're still alive is also the reason why the Bitcoin Blockchain is still alive." ${ }^{33}$ Eventually, the social contract as incorporated in Ethereum is seen as a game theoretical mechanism that underlies all social interactions and only needs to be "facilitated" by blockchain technologies. This assumed that game theory can thus correctly predict human behavior as it "really" is and that this knowledge can be used to "engineer" social interaction in a virtual environment that functions like a game environment.

Our initial conclusions support the view that the justification offered for blockchain governance to a certain extent resembles justification accounts offered by social contract theories. It is most similar to the justification of the social contract presented by Hobbes, in that it is based on a rather negative assessment of human nature, being self-interested and potentially corrupt, and tends to reduce social interactions to game-theoretical problems. In contrast, the initial situation it presents resembles the scheme presented by Rousseau, in that the undesirable "pre-blockchain" society is defined by our institutional reality rather than by a state of nature lacking any form of government. Finally, we argue that blockchain governance seems to approximate Rawls's original position, although it makes this position unattainable by rendering inequality between contracting parties a structural feature of the technology.

\section{Modeling Sovereignty in Blockchain Governance}

Having examined the theme of governance justification, we now examine models of governance, or more specifically identify ways in which the models of governance presented by blockchain technologies reflect aspects of the models of governance presented by social contract theories. By doing so, we do not intend to provide an account of how blockchain government actually works, for such an account would be highly speculative in the current state of affairs in which no instance of wholly functioning blockchain governance exists, but rather of similarities between models of governance as they are being claimed to manifest themselves through the use of blockchain technologies and those discussed by social contract 
theories. A central notion in social contract theories specified as a solution to the problem of the initial situation is the notion of "sovereignty." This section will focus on this notion, examining the views of Hobbes, Rousseau and Rawls on the issue of sovereignty. In contrast to the previous section, in which our analysis relied on linking ideas from key philosophical texts with the views on justification of blockchain governance expressed by the blockchain community, we now develop our comparison with a focus on the core design features of the technology for our analysis.

Hobbes views the creation of an absolute form of government, which he designates as the "Leviathan," as the only rational way people could escape the miseries of their state of nature. By contracting together, people alienate all their rights to the Leviathan, which can be viewed as the sovereign power (such as a monarch) in abstract. Hobbes describes the Leviathan as a "real Unitie of them all, in one and the same Person, made by Covenant of every man with every man ... this is the Generation of that great Leviathan, or rather (to speake more reverently) of that Mortall God" (1651: 227). The Leviathan is where sovereignty - supreme authority - resides; and all people, having alienated their rights to the sovereign, are obligated to obey its decrees. Hobbes argues that the sovereign (be it one person or an assembly) has power over everyone else - all of whom are subjects - and "to the end he may use the strength and means of them all, as he shall think expedient, for their Peace and Common Defence" (1651: 228). The Leviathan is the sovereign, and once created it is totalitarian, despite having been created voluntarily by its subjects. Attaining sovereign power, Hobbes argues, occurs "when men agree amongst themselves, to submit to some Man, or Assembly of men, voluntarily, on confidence to be protected by him against all others. This latter, may be called a Political Common-wealth, or Commonwealth by Institution..." (1651: 228). The only rights that people have within such a commonwealth by institution are those granted to them by the sovereign, with the significant exception of the right to self-preservation. The Leviathan, as the absolute sovereign, cannot be questioned and must be obeyed; otherwise people have to face the threat of inevitable punishment.

Rousseau's notion of the sovereign is in some ways similar to the view expressed by Hobbes. Rousseau suggests that the clauses of the social contract can be summarized as "the total alienation of each associate, together with all his rights, to the whole community; for, in the first place, as each gives himself absolutely, the conditions are the same for all; and, this being so, no one has any interest in making them burdensome to others" (1762: 191). Unlike Hobbes, however, Rousseau argues that "each man, in giving himself to all, gives himself to nobody; and as there is no associate over which he does not acquire the same right as he yields others over himself, he gains an equivalent for everything he loses, and an increase of force for the preservation of what he has" (1762: 192). In this way, if all associates agree on instituting a regime of property rights that applies the same conditions on all, no associate will defect from it. This is because anyone defecting from the agreement will, in addition, lose their property rights. Moreover, for Rousseau, the individual does not alienate her freedom when entering the social contract in the way that the individual for Hobbes does but rather voluntarily cooperates with others in order to increase her freedom while being still involved in the creation of laws and rules governing her life. For Rousseau, each individual has put "his person and all his power under the supreme direction of the general will, and, in our corporate capacity, we receive each member as an indivisible part of the whole" (1762: 192). Each person then, in uniting with others "may still obey himself alone, and remain as free as before" (1762: 191). This freedom is due to the fact that, for Rousseau, sovereignty can never be alienated from the individuals forming the society and, as such, sovereignty resides not 
principally in a centralized assembly or monarch (as it does for Hobbes), but is always vested in the will of the people - in a decentralized manner. Rousseau considered that whilst assemblies or monarchs might attempt to usurp power, this is always illegitimate, for the sovereignty of the people is inalienable. Sovereignty, for Rousseau, is something that exists in and for all people who have taken part in the social contract. In other words, it does not reside in a central sovereign authority but rather decentralized in the agency of each member of a community. Therefore, Rousseau prefers a form of direct democracy (one man, one vote) as a model of governance and a high level of transparency of decision making for any type of representational governance, so that representatives can always be subjected to public scrutiny (Inston, 2010: 152).

The model of governance proposed by Rawls is more abstract compared to those of Hobbes and Rousseau, in that it does not propose a specific type of authoritarian or democratic rule (though Rawls is a strong supporter of democratic institutions) but rather a social contract conditioned by certain "principles of justice." Rawls proposes two principles of justice that every contracting individual behind the "veil of ignorance" would rationally consent to (Rawls 1971: 53):

(1) "Each person is to have an equal right to the most extensive total system of equal basic liberties compatible with a similar system of liberty for all"

"Social and economic inequalities are to be arranged so that they are both (a) reasonably expected to be to everyone's advantage, and (b) attached to positions and offices open to all"

Thus, every model of governance should, according to Rawls, incorporate these principles in order to be justifiable. However, he also concedes that any sovereign should provide for a publicly maintained, effective schedule of penalties, "so men in the absence of coercive arrangements establish and stabilize their private ventures by giving one another their word" (1971: 305). Thereby, the sovereign makes sure that people reciprocally recognize promises made to one-another that are based on common knowledge i.e. the conditions of these promises should be publicly identified.

The model of governance offered by the Ethereum platform is perhaps best described by Binmore, who states that "a social contract is"..."an equilibrium profile of strategies, one for each citizen. When the social contract operates, each citizen will therefore be optimizing when he follows the rules of behavior prescribed by his strategy" (1998: 355). ${ }^{34}$ A blockchain technology such as Ethereum can be said to provide its users with an "equilibrium profile of strategies" that are hard-coded in the blockchain protocol. Within this equilibrium profile, participants interact and are consenting by default with the agreed upon rules in a particular smart contract; however, the limits of what kind of smart contracts could run on the Ethereum protocol are still unclear. The Ethereum Wiki page claims: "ultimately, Ethereum could be used to run countries." ${ }^{35}$ Gavin Wood, a co-founder of Ethereum, sees the importance of the emerging and voluntary status of the social contract in shaping social interaction and a significant force in human cooperation: [Ethereum's use of blockchain technologies demonstrates that] "through the power of the default, consensus mechanisms and voluntary respect of the social contract, it is possible to use the internet to make a decentralized valuetransfer system, shared across the world and virtually free to use." ${ }^{\text {, }}$

To examine the extent to which conceptions of sovereignty in blockchain governance reflect the ideas of sovereignty discussed by social contract theories we first consider the Leviathan, as presented by Hobbes, as a model of governance. Even though Hobbes and Nakamoto foresee different roles for the sovereign in their writings (understood respectively 
as the Leviathan and the consensus mechanism), there are striking similarities as well. Within a single blockchain, disobeying the rules is made impossible and will lead to exclusion from the system - i.e. the blockchain is totalitarian in terms of rule-enforcement, which makes it comparable to the Leviathan as described by Hobbes. Moreover, no blockchain can be altered or manipulated by the individuals who use it to contract with one-another. In order to render fraud and counterfeit structurally impossible, once a person has contracted with someone else through the blockchain she has no other choice than to abide by its rules. Important to note, however, is that this structural impossibility only exists within the system that runs on the blockchain. Participants running the software can circumvent it by not using a certain blockchain technology or by switching between different blockchain technologies.

As Rawls (1971: 453) concedes, the sovereign for Hobbes is a mechanism that stabilizes a system of human cooperation. Similarly, the blockchain can be understood as a mechanism for stabilizing a pre-given system of human cooperation such as a property regime or an insurance system. Any blockchain can therefore be seen as a created "institution", a technological Leviathan (or "techno-leviathan" as expressed by Brett Scott) ${ }^{37}$ that people voluntarily join. As a counterpoint to the totality of power assigned to the Leviathan for Hobbes, blockchain governance is not "absolute," in the sense that no blockchain dominates the entire governance of a community, and as such it is unable to realize the ideal of the Leviathan expressed by Hobbes. In contrast to the Leviathan, the blockchain does not have the power or authority to kill those who use it to contract with one-another and it cannot change its rule according to its own will.

Hobbes argues that the Leviathan's power is sustained by means of a constant threat of punishment whenever its subjects act against its decrees, raising the issue of whether blockchain governance establishes any such system of punishment. There are some suggestions in the literature, for example Chuen argues, in discussing the role of the social contract for blockchain technologies: "by social contract, we mean a system for which to be part of it means obeying the rules." ${ }^{38}$ These rules, however, are not enforced "under the threat of physical action or exclusion ... but on the blockchain, the rules cannot be broken and so exclusion is implicit" (Chuen, 2015: 391). Thus, enforcement of the social contract by means of blockchain technologies differs from the Hobbesian idea of enforcement by threat of physical punishment. The majority of nodes within the system act as the sovereign by enforcing its rules on all of its participants. This design feature of the blockchain brings us to Rousseau's version of social contract theory.

Rousseau insisted that "in order that the social contract may not be an empty formula, it tacitly includes the undertaking, which alone can give force to the rest, that whoever refuses to obey the general will, shall be compelled to do so by the whole body," or infamously "this means nothing less than that he will be forced to be free" (1762: 195). Similarly, the consensus mechanism built into blockchain technologies ensures that those interacting through a blockchain application are compelled to abide by its rules. In an illuminating presentation, Buterin explains that decentralized communities using a blockchain technology will instantiate "recursive punishment" systems. ${ }^{39}$ This implies that, although a node controlled by a miner is free to go against the "general will" of the blockchain, it is deterred from doing so because both this node and other nodes following the same strategy will eventually be punished by being excluded from the system; or more precisely by being excluded from the main blockchain and working on another chain that represents no value. The question of course is whether implicit exclusion from a blockchain is a sufficient deterrent to ensure that all its members always obey its rules at all times. The point can be 
addressed with reference to the extent to which one blockchain dominates one or more aspects of social life. A simple illustration of this is to imagine if property rights in the context of the Internet of Things (IoT) were to be organized through one dominant blockchain application. Exclusion from this blockchain would mean that the physical devices owned by an excluded individual could cease to function and thus the punishment of exclusion would be sufficiently serious to deter people from individually contravening the rules laid down by the blockchain.

In addition to matter of rule compliance, blockchain governance also reflects Rousseau's idea of sovereignty, at least to a greater extent than the highly centralized idea of sovereignty expressed by Hobbes. Similar to Rousseau's ideal of radical democracy, sovereignty on the blockchain is implemented in a decentralized manner: all the nodes together enforce the validity of transactions and therefore reflect consensus with regards to the contractual agreements realized through the blockchain. In theory at least, Rousseau's ideal of a general assembly that encompasses all the members of a community could be technically realized in blockchain governance. All members of a blockchain community could be permitted to propose their own smart contracts and vote on contracts proposed by others.

There is, however, a significant difference between Rousseau's concept of the General Will and sovereignty in blockchain governance, which in many ways represents instead the "will of all." The General Will, in Rousseau's conception, is primarily concerned with the common interest, in contrast with the "will of all" as implemented in blockchain governance, which is no more than the sum of the individual wills of its members. The blockchain design lacks any conception of a common interest beyond facilitating autonomous individuals contracting between themselves. The blockchain then, is based on a limited conception of the "common good," one that is more consistent with the ideals of contemporary capitalism, than the Republican ideals of Rousseau. Rousseau also provides a warning regarding the distribution of power in contract-based political organization that remains pertinent to blockchain technologies. These technologies instantiate distributed networks, that can theoretically be comprised of all those who participate in them. The power resides with those who control the nodes, ensuring that there can in theory be no central power or authority as long as a sufficient number of non-related nodes partake in the network. Arguably then, within the blockchain, sovereignty is distributed at the technological level, rather than explicitly at the political level. In principle, it is possible for the miners to unite and gain control of the blockchain, similar to the risk of elected representatives attempting to usurp sovereignty and limit it only to themselves, as foreseen by Rousseau. Such a concern is raised in current debates on the "centralization" of Bitcoin; which focus on the risks of pools of miners coordinating their mining efforts to undermine the system. ${ }^{40}$

There seems to be no guarantee that all subjects of a hypothetical blockchain government would act under the condition that Rousseau portrayed as "freedom and equality of all" (Inston 2010: 175). This concern can be addressed with reference to Rawls's idea of sovereignty. Blockchain governance seems to have the capacity to support Rawls's first principle of justice, since people contracting through the blockchain would all enjoy the same rights and liberties. The blockchain does not discriminate against its users based on who they are, and as such, in theory all users are able to contract with one-another while enjoying the same, though limited, digital rights and liberties, such as the right to smart property or the right to freedom of expression on the blockchain. Rawls's second principle of justice seems, however, to be very hard - if not impossible - to realize in blockchain governance. In accordance with the libertarian ideas that support blockchain governance, such governance seems to be designed to exclude hardcoded ideas of distributed justice. Firstly, there are no 
political offices "open to all" in blockchain governance able to intervene in the way rights and assets are distributed amongst its members. Nobody is able to superimpose a redistribution of rights and assets because the only distribution that is structurally enabled in blockchain governance is the one that happens to be the equilibrium resulting from the interacting nodes. Moreover, no limitations exist for great inequalities in distribution of rights and assets, especially because individuals or companies can own multiple nodes in the system.

This last point has been made strikingly clear in the aftermath of the recent "DAO attack." "The DAO" is a project that runs on the Ethereum protocol but is a separate initiative that can be seen as the first high-profile implementation of the idea of a Decentralized Autonomous Organization. Individuals are able to arrange smart contracts in the DAO and join them by pledging "DAO tokens" that can also be used to vote for proposals that designate how the tokens belonging to a smart contract should be spent. By exploiting a bug in the source code of the DAO, an attacker managed to obtain an equivalent of 60 million USD in the cryptocurrency Ether. ${ }^{41}$ We will not discuss the technical details of this attack, but focus instead on the "ideological" conflict it created in the Ethereum community. Although the cryptocurrency was obtained by exploiting a weakness in the source code, the attacker obtained the Ether "legally" within the system (recall the earlier discussion that a blockchain can be considered as a "form of law"). The response of the Ethereum community was split, with some members arguing that the attacker should be allowed to keep his "reward" and that the software actually worked as it was intended to, while other members argue that the basic code of the DAO should be rewritten to prevent the attacker from claiming the Ether obtained in the attack.

This division within the community illustrates a tension concerning the justifiability of existing governance models. The argument remains that sovereignty resides in the blockchain, that the mechanisms of interaction that existed at the moment when people consented to abide to the internal rules of the DAO are the only ones that should validate transactions. This perspective is, though, in opposition to the widely held view that the distribution of Ether after the attack is unfair and that the Ether should be redistributed by means of a "hard fork" that would in effect circumvent the sovereignty of the current blockchain. A Rawlsian argument could be constructed to support this latter argument. Behind a "veil of ignorance" in which nobody knows their position (including the attacker), the preference of the least advantaged (the individual losers from the attack) would be endorsed. A particularly compelling argument can be made on the basis that the attacker is the sole beneficiary, while the losing parties are not merely those losing part of their investment, but the entire network because the DAO as a whole lost value due to the attack. This conflict raises the issue of whether a blockchain technology such as the DAO can offer a justifiable model of governance while lacking an external governance structure to function as a check on the power of the technology. As Yarvin argues: "one of the governance problems of blockchains, related to the fundamental error of decentralization theater, is the failure to build deliberative institutions on top of the "parliament of miners.",42 While the DAO in question was relatively small in both scale and scope, with few contracts in operation at the moment of the attack, if in the future governance of crucial parts of our social infrastructure, such as identity registers or property rights, were to be organized in the form of DAOs, these conflicts might cause great social unrest, rebellion and possible challenges concerning the sovereignty of the blockchain. This illustrates clearly that issues regarding how to model governance on the blockchain, and how to govern the blockchain itself, have yet to be resolved and might yet become relevant research topics in political philosophy and political issues in their own right. 


\section{Conclusion}

In this paper, we investigated the way in which the justification and modeling of blockchain governance can be said to reflect core ideas in social contract theories. The following are our main findings:

- Accounts of justification of blockchain governance are informed by a conception of human nature that is similar to the account offered by Hobbes; however, it is similar to Rousseau's justification of governance in that it is seen as a solution to an existing structure of corrupted institutions.

- Blockchain governance in many ways reflects Rawls's idea of a "veil of ignorance," being non-discriminatory, though it negates this idea because power-relations are predefined in the public ledger.

- The blockchain reflects the idea expressed by Hobbes of a totalitarian sovereign in terms of rule-enforcement, coupled with Rousseau's idea of decentralized governance and Rawls's idea of equal rights and liberties for all (that is, for all the nodes).

- Blockchain governance fails, however, to incorporate Rousseau's idea of the common good, and fails to implement conditions of distributive justice that Rawls thought to be essential for overcoming the initial situation.

A first implication of our discussion has been to contest the idea that the blockchain is a "neutral," non-political technology. Instead, being a transformative technology, its political implications are significant because the applications that the technology affords can reconfigure economic, legal, institutional, monetary and ultimately broader socio-political relationships. ${ }^{43}$ By discussing the blockchain in light of social contract theories, we have tried to make explicit what kind of political justifications for blockchain governance are offered and what political model of governance it represents.

Overall, it seems that the justification and modeling of governance presented by Hobbes, though far removed from anarchist and libertarian ideals that fuel many of the efforts for designing blockchain technologies, offers an insightful comparison with blockchain governance. The justification of blockchain governance on the basis of a negative view of human nature and game-theoretical presuppositions, and its modeling as a totalitarian process in the sense that its authority is unquestionable once voluntarily joined, brings it surprisingly close to the social contract theory expressed by Hobbes. Although Rousseau's model of governance offers some striking similarities with blockchain governance, based on his focus on decentralization of power and punishment through exclusion, Rousseau's ideas of governance in support of the common good and governance based on free and equal participation of community members seem to be lacking in blockchain governance. In a more radical reading, it could be argued that Rousseau denounces any delegation of governance to a technology when he stresses: "The general will is ultimately unrepresentable because it entails a continuous act of willing which leaves its identity forever incomplete and thus available to new demands and reformulations" (Inston 2010: 130). Thus, any technology instantiating human governance along fixed lines would be essentially inadequate. Finally, Rawls's social contract theory seems to show only limited similarities with blockchain governance. Although a blockchain might seem to offer a limited form of a "veil of ignorance" for people contracting through it, it lacks the essential elements of distributive justice that would make it a justifiable form of governance in Rawls's terms. 
While we feel these conclusions are insightful, and appropriately evidenced, a number of important limitations of our inquiry are worthy of mentioning. Firstly, our discussion of social contract theories has been necessarily incomplete, both by only addressing three of their prominent instantiations but also by discussing only a limited number of their central aspects (focusing on their notions of the initial situations and sovereignty, and thereby leaving out discussions of issues such as transparency and consent). Secondly, we have only focused on a limited number of blockchain technologies, notably on Ethereum, omitting from our analysis interesting examples such as Bitnation that might have influenced parts of the argument. ${ }^{44}$ Thirdly, and perhaps most importantly, our analysis is based on a technology that is still in its development phase, which means that empirical support for much of our discussions is lacking or in its infancy. In the future blockchain technologies might be developed in ways that we have failed anticipate in this paper, which resolve the governance dilemma, such as providing mechanisms of distributive justice, for example. Therefore, our paper should be seen as an exploration of the potential implications of blockchain governance and in providing the scope for future research on this topic in the field of political philosophy.

\section{Acknowledgements}

The ADAPT Centre for Digital Content Technology is funded under the SFI Research Centres Programme (Grant 13/RC/2106) and is co-funded under the European Regional Development Fund.

\section{Author Contributions}

WR provided the core insights about the elements of social contract theories relevant to our investigation (40\%), FOB interpreted these insights in relation to the core design features of blockchain technologies (30\%) and $\mathrm{PH}$ added to the paper by incorporating the views of the key Ethereum community members $(30 \%)$. All equally contributed to manuscript preparation.

\section{Notes and References}

\footnotetext{
${ }^{1}$ Karlstrøm, H. "Do libertarians dream of electric coins? The material embeddedness of Bitcoin." Distinktion: Scandinavian Journal of Social Theory 15.129 (2014) http://doi.org/10.1080/1600910X.2013.870083

2 Swan, M. Blockchain: Blueprint for a New Economy Sebastopol: O'Reilly Media Inc (2015)

${ }^{3}$ Kostakis, V., Giotitsas, C. "The (A)Political Economy of Bitcoin.” tripleC: Journal for a Global Sustainable Information Society 12.2 431-440 (2014)

${ }^{4}$ Barton, P. Bitcoin and the Politics of Distributed Trust (Senior Thesis). Swarthmore College
} (2015)

${ }^{5}$ Golumbia, D. "Bitcoin as Politics: Distributed Right-Wing Extremism." In Moneylab Reader: An Intervention in Digital Economy. Amsterdam: Institute of Network Cultures 117-131 (2015)

${ }^{6}$ DuPont, Q. "The Politics of Cryptography: Bitcoin and The Ordering Machines." Journal of Peer Production 1.4 1-10 (2014) 
${ }^{7}$ Kavanagh, D., Miscione, G. "Bitcoin and the Blockchain: A coup d'état in Digital Heterotopia?" In Critical Management Studies Conference. Leicester (2015)

${ }^{8}$ Dupont, Q., Maurer, B. "Ledgers and Law in the Blockchain." Kings Review (23 June 2015) http://kingsreview.co.uk/magazine/blog/2015/06/23/ledgers-andlaw-in-the-blockchain/

9 Ethereum. "About the Ethereum Foundation" (accessed 25 January 2016) https://www.ethereum.org/foundation

${ }^{10}$ Wood, G. "Ethereum: A Secure Decentralised Generalised Transaction Ledger," Ethereum Project Yellow Paper. Gavwood (accessed 28 November 2015) http: //gavwood.com/Paper.pdf

${ }^{11}$ Alexander, R. "The First Blockchain Wedding." Bitcoinmagazine (accessed 3 December 2015) https://bitcoinmagazine.com/articles/first-blockchain-wedding-21412544247

${ }^{12}$ Buterin, V. "A next-generation smart contract and decentralized application platform." Ethereum 1-36 (2014) http: / / buyxpr.com/build/pdfs /EthereumWhitePaper .pdf

${ }^{13}$ Wright, A., De Filippi, P. "Decentralized Blockchain Technology and the Rise of Lex Cryptographia." Social Science Research Network 2580664 (2015)

http: / / papers.ssrn.com/abstract $=2580664$

${ }^{14}$ Ethereum. "What is Ethereum?" Etherscripter (accessed December 3 2015) http://etherscripter.com/what_is_ethereum.html

${ }^{15}$ Buterin, V. "DAOs, DACs, DAs and More: An Incomplete Terminology Guide." Ethereum (accessed 12 July 2016) https : / blog.ethereum.org/2014/05/06/daos-dacsdas-and-more-an-incomplete-terminology-guide/

${ }^{16}$ Skyrms, B. Evolution of the Social Contract. Cambridge: Cambridge University Press (1996)

${ }^{17}$ Hobbes, T. Leviathan. London: Andrew Crooke (1651)

${ }^{18}$ Rousseau, J.-J. The Social Contract and Discourses. London: Everyman (1762)

${ }^{19}$ Rawls, J. A Theory of Justice. Cambridge, Massachusetts: Harvard University Press (1971)

${ }^{20}$ Ihde. D. Postphenomenology and Technoscience. New York: Sunny Press (2009)

${ }^{21}$ Winner, L. "Do artifacts have politics?” Daedalus 109.1 121-136 (1980)

${ }^{22}$ Simmel, G. The Philosophy of Money ( $3^{\text {rd }}$ ed.). New York: Routledge Classics (1900)

${ }^{23}$ Dodd, N. “On Simmel's Pure Concept of Money: a Response to Ingham.” European Journal of Sociology 48.2 273-294 (2007)

${ }^{24}$ Chung, H. "Hobbes's State of Nature: A Modern Bayesian Game-Theoretic Analysis." Journal of the American Philosophical Association 1.3 485-508 (2015) 
LEDGER VOL 1 (2016) 134-151

${ }^{25}$ Inston, K. Rousseau and Radical Democracy. London: Continuum International Publishing Group (2010)

${ }^{26}$ Nozick, R. Anarchy, State and Utopia. Oxford: Blackwell Publishing (1974)

${ }^{27}$ Simon, Y., Kuic, V. “A Note on Proudhon’s Federalism.” Publius 3.1 19-30 (1973)

${ }^{28}$ Buterin, V. "A Next-Generation Smart Contract and Decentralized Application Platform." Ethereum (2013) https: //www.ethereum.org/pdfs/EthereumWhitePaper.pdf

${ }^{29}$ Roio, D.J. "Bitcoin, the End of the Taboo on Money." Dyne.org digital press (April 2013) https://files.dyne.org/readers/Bitcoin_end_of_taboo_on_money.pdf

${ }^{30}$ Schejter, A. \& Yemini, M. "Justice, and Only Justice, You Shall Pursue: Network Neutrality, the First Amendment and John Rawls's Theory of Justice." Michigan Telecommunications and Technology Law Review 14.1 137-174 (2007).

${ }^{31}$ O’Dwyer, R. "The Revolution will (not) be Decentralized: Blockchains." Commons Transition (2015)

${ }^{32}$ Nakamoto, S. "Bitcoin: A Peer-to-Peer Electronic Cash System.” No publisher. 1 (2008) https://bitcoin.org/bitcoin.pdf

${ }^{33}$ Buterin, V. "Vitalik Buterin: Cryptoeconomic Protocols In the Context of Wider Society." Youtube (accessed 26 January 2016) https: / /www.youtube.com/watch?v=S4 7 iWiKKvLA

${ }^{34}$ Binmore, K. Game Theory and the Social Contract (3rd ed.) Cambridge, Massachusetts: Massachusetts Institute of Technology 355 (1998)

${ }^{35}$ No Author. "Ethereum wiki." Github (accessed 28 November 2015) https://github.com/ethereum/wiki/wiki

${ }^{36}$ Wood, G. "Ethereum: A Secure Decentralised Generalised Transaction Ledger." Gavwood (accessed 28 November 2015) http: //gavwood.com/Paper.pdf

${ }^{37}$ Scott, B. "Visions of a Techno-Leviathan: The Politics of the Bitcoin Blockchain." EInternational Relations (accessed 16 July 2016) http: / /www.eir.info/2014/06/01/visions-of-a-techno-leviathan-the-politics-ofthe-bitcoin-blockchain/

${ }^{38}$ Chuen, D. L. K. Handbook of digital currency: Bitcoin, Innovation, Financial instruments, and Big Data. Handbook of Digital Currency. London: Elsevier Inc. (2015) http://doi.org/10.1016/B978-0-12-802117-0.09989-6

${ }^{39}$ Buterin, V. "Vitalik Buterin: Cryptoeconomic Protocols In the Context of Wider Society." Youtube (accessed 26 January 2016) https://www. youtube.com/watch?v=S4 7 iWiKKvLA 
${ }^{40}$ Fargo, S. "The economics of Bitcoin Mining." Insidebitcoins.com (accessed 26 January 2016) http: //insidebitcoins.com/news/the-economics-of-bitcoin-miningcentralization/31833

${ }^{41}$ Reutzel, B. "The DAO Shows Blockchain Can't Code Away Social Problems." Coindesk (accessed 18 July 2016) http: / /www.coindesk.com/system-problems-socialissues-daos-structure/

${ }^{42}$ Yarvin, C. "The DAO as a lesson in decentralized governance." Urbit (accessed 18 July 2016) https://urbit.org/blog/dao/

${ }^{43}$ Coeckelbergh, M., Reijers, W. "Crypto currencies as narrative technologies." SIGCAS Computers \& Society, 45.3 172-178 (2015)

${ }^{44}$ Bitnation. "Governance 2.0: borderless, decentralized, voluntary." Bitnation (accessed 18 July 2016) https://bitnation.co/main/

(cc) BY ULLS
Articles in this journal are licensed under a Creative Commons Attribution 4.0 License.

Ledger is published by the University Library System of the University of Pittsburgh as part of its D-Scribe Digital Publishing Program and is cosponsored by the University of Pittsburgh Press. 